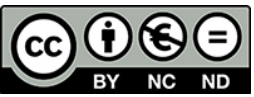

\title{
Approches méthodologiques et expérimentales des phénomènes complexes.
}

\author{
Gilbert BELAUBRE \\ gilbert.belaubre@polytechnique.org \\ Académie Européenne Interdisciplinaire des Sciences AEIS, Paris, France, Europe, \\ http://www.science-inter.com \\ Association Française de Science des Systèmes AFSCET, Paris, France, Europe \\ http://www.afscet.asso.fr
}

\begin{abstract}
Résumé - La complexité de la nature est multiforme, et il est nécessaire de mettre en oeuvre des approches méthodologiques variées pour tenir compte de cette variété. Les efforts de représentation et d'explication des phénomènes naturels nous conduisent toujours à rechercher, en priorité, des relations de proportionnalité entre les mesures de ce que nous considérons comme des variables pertinentes. La linéarité a été et reste la règle d'or de nos représentations. Mais beaucoup de phénomènes sont rebelles à cette réduction. Les ordinateurs nous aident aujourd'hui à intégrer des myriades de données et à bâtir des modèles non linéaires. Ils ne se substituent pas à la pensée heuristique du savant, mais ils allègent les calculs et permettent des tests d'hypothèses quasiment instantanés. Il a fallu de longs mois de calcul pour que Le Verrier définisse l'orbite d'Uranus. Aujourd'hui, les ordinateurs exécutent ces calculs en quelques microsecondes, et ils ne font pas d'erreurs. Les complexités auxquelles nous devons faire face sont de plusieurs ordres : 1- celles de la physique statistique et de l'astrophysique, 2 - celle des constructions moléculaires complexes, qui sont les précurseurs des organismes vivants, 3 celle des morphogenèses et des embryogenèses, 4- celle de la complexité neuronale, de ses processus que nous appelons le mental, 5- celles des phénomènes sociaux chez les animaux inférieurs, 6 - celle des animaux sociaux, y compris les humains, particulièrement compliqués par leurs capacité d'anticipation, donc d'interactions réciproques.
\end{abstract}

Mots clés : biologie, complexité, démographie, échelle, économie, émergence, évolution, fractale, management, modèle, morphogenèse, réseaux, systèmes sociaux

\section{INTRODUCTION}

Beaucoup de ces recherches, en cosmologie et dans le vivant, rencontrent des obstacles idéologiques hérités des mythologies, animisme, paganismes ou religions. Les questions relevant de la physique statistique et de l'astrophysique sont désormais en charge de méthodologies éprouvées. Celles concernant l'activité mentale ne pourront être abordées que sur des bases solides de la biologie et des morphogenèses spécifiques des systèmes nerveux centraux.. Toutes les recherches en cours vont dans ce sens. Compte tenu du thème général de ce colloque, je me propose d'aborder, très sommairement, les questions que soulèvent les processus biologiques et les morphogenèses, et de présenter plus précisément celles concernant les recherches en modélisation des comportements humains, individuels et sociaux.. Les prémisses de ces comportements chez les animaux dits «supérieurs » pourront être évoqués. J'aborderai donc les recherches en modélisation dans les domaines de la démographie, des mouvements de populations, des développements urbains, des phénomènes économiques et financiers, et enfin, les questions relatives à la gestion et plus généralement à la gouvernance.

Je renvoie en Annexes quelques développements qui alourdiraient ma présentation, mais qui apportent un certain éclairage indispensable pour donner une orientation globale à ma présentation :

La question de la double représentation probabiliste et déterministe des phénomènes moléculaires en biologie.

Les travaux d'élucidation des phénomènes de transition (émergences et des morphogenèses.

L'évolution des modélisations depuis un siècle.

Développement des performances cognitives au cours de l'évolution.

Une étude critique de trois types successifs de modélisations sur un exemple très éloquent en démographie. Un article que je dois à Daniel Courgeau [7]. 


\section{QUESTIONS}

\section{$1^{\text {ĖRE }}$ QUESTION : COMMENT LA COMPLEXITÉ BIOLOGIQUE SE PRODUIT-ELLE ?}

Les conditions d'apparition des molécules complexes dépendent d'abord de la présence des corps simples qui les composent. C'est là un point auquel l'astrophysique répond. En deuxième lieu, les structurations moléculaires sont régies par la grande variété des liaisons électroniques qui sont de l'adhésion électrostatique (forces de Van der Waals à la covalence (diamant). Ces liaisons sont largement favorisées par l'eau. En effet le dipôle de la molécule d'eau favorise l'ionisation, et les liaisons ioniques qui en résultent sont d'une grande variété et elles provoquent des assemblages moléculaires stables. La diversité et la complexité biologique sur terre est sûrement la cause décisive de l'apparition de la vie, et l'eau y a été indispensable.

Depuis la célèbre expérience de Miller en 1953, qui a fabriqué des acides aminés dans une atmosphère sillonnée d'éclairs (décharges électriques) tous les laboratoires du monde fabriquent des molécules prébiotiques, mais certains, comme Di Mauro [11], à l'Université La Sapienza, à Rome, fabriquent des acides nucléiques et des acides gras qui sont à la base de la formation des membranes cellulaires. Par manipulation, on peut fabriquer de l'ADN. L'expérience de John Venter en 2010 crée une cellule à génome synthétique. On peut fabriquer des membranes. On peut penser que des êtres vivants pourront être fabriqués en laboratoire, avant même qu'on n'ait élucidé l'origine de la vie sur terre, cette dernière perspective étant jugée inadmissible par une fraction non négligeable de l'humanité.

Il y a, actuellement, peu d'éléments pour comprendre le processus qui a conduit à la vie sur terre. . Les moyens techniques dont disposent les chercheurs sont strictement adaptés au but poursuivi . La nature ne fonctionne pas de cette manière, c'est du moins la conviction des scientifiques depuis que Darwin [10] a apporté une masse de preuves qui m'ont conduit à formuler la théorie de l'évolution. Dans son principe, , cette théorie se fonde sur « le hasard suivi de la sélection naturelle. On peut dire aussi « des concours de circonstances avec stabilisation dynamique ».

Ces notions renvoient à une interprétation probabiliste. Or, le déterminisme génétique s'oppose à cette interprétation. Il faut voir là une des nombreuses alternances dont l'histoire de a science nous offre maints exemples. Des théories et des modèles opposés peuvent s'affronter jusqu'à ce qu'un éclaircissement arrive. Je donne en Annexe 1 quelques éléments sur ce sujet crucial.

\section{2ÈME QUESTION : QUELS SONT LES MÉCANISMES DES MORPHOGENÈSES ?}

Ces développements sont étudiés dans les domaines de la physique, en cristallographie, où Pierre Curie [9] s'est illustré en fondant la notion générale de symétrie comme un principe majeur de la physique; René Thom, partant des formes de la géométrie, a voulu les impliquer dans les morphogenèses de la nature ; d'autres, comme Denis Noble [24], ont étudié les structures du vivant et leurs évolutions en mettant en jeu les variables qui leur paraissaient pertinentes avec les outils des mathématiques. Cette voie pragmatique donne déjà d'incontestables résultats. Enfin, John Holland [16] a étayé par des exemples très variés le principe de la morphogenèse comme processus de génération sous contrainte. Cette vision englobe les phénomènes d'interactions entre phases (la plupart des phénomènes fractals [12]), les émergences en tant que processus de transition entre niveaux de structuration enfin et surtout, les embryogenèses comme morphogenèse fondamentale des êtres vivants multicellulaires.

Des précisions sur le décryptage des morphogenèses et des émergences sont données dans l'Annexe 2. Ces interprétations sont fondamentalement causales, sinon déterministes, au sens que j'évoque dans l'Annexe 1 .

Mais : quid de la complexité, quand le libre arbitre s'en mêle ? Il est clair qu'il y a une rupture dans le niveau de complexité lorsqu'on aborde le comportement des êtres vivants dotés d'un système nerveux central. Il est indubitable, aujourd'hui, que ces êtres disposent, même à un degré rudimentaire, d'une capacité de représentation de leur environnement. Lorsque les perceptions deviennent conscientes, l'individu apparait, et dans un stade encore supérieur de l'individuation, il devient autonome. Dès lors, les agrégats d'individus ne peuvent plus être considérés comme des structures liant strictement leurs éléments., et les modèles empruntant cette voie échouent. Nous le verrons dans un exemple développé dans l'Annexe 5. L'étude des sociétés animales (mammifères), et humaines, appelle donc des modèles d'interaction entre individus et « corps social. Ceci nous conduit à la troisième question. 


\section{3ÈME QUESTION, D'ORDRE MÉTHODOLOGIQUE : COMMENT ABORDER LA REPRÉSENTATION THÉORIQUE DE LA COMPLEXITÉ DES SYSTÈMES SOCIAUX ?}

Les sciences sociales se développent, aujourd'hui, sur les principes d'induction rationnelle (Franck [14]) de la physique statistique et de la biologie. Mais leurs modèles doivent prendre en compte une complexité d'un ordre supérieur. La plupart des thèmes de ce colloque concernent des sujets centrés sur le comportement humain. Ce comportement s'insère dans une évolution dont on trouve les prémisses chez les animaux. L'éthologie animale nous aide à bâtir un continuum dans l'évolution des performances intellectuelles. Je donne en Annexe 4 quelques éléments de base concernant l'évolution, afin d'en marquer les étapes cruciales qui appellent des modélisations adaptées.

\section{Les modèles dans les sciences sociales}

Je vais, dans la grande variété des études récentes, présenter une aussi grande variété de modèles. Il s'agit le plus souvent, de «modèles à base d'agents", les agents étant les unités actives dans les phénomènes étudiés. Les agents sont définis par leurs caractéristiques et leurs propriétés, par celles du moins qu'il est indispensable de prendre en compte dans l'étude. Les propriétés des éléments et leurs interactions définissent le processus, mais le choix du modèle (ou de la théorie), appartient au chercheur. On trouvera en Annexe 3 une revue rapide des types de modélisations inventés au cours du $20^{\text {ème }}$ siècle. Atlan situe la complexité « entre le cristal et la fumée ». La notion de modèles à base d'agents nous permet d'aller plus loin dans la typologie des niveaux de complexité. Bien entendu, le titre d'Atlan est une image. La complexité du cristal est faible par rapport à celle d'une morphogenèse. Et dans les morphogenèses, le degré de complexité dépend de l'intensité des liens qui unissent les éléments.

Les structurations neuronales, mais aussi les structurations des groupes d'individus animaux, et surtout humains, présentent des dynamiques particulièrement complexes lorsqu'elles se forment en boucles de rétroactions.

\section{Réseaux neuronaux}

Des expériences menées initialement par Singer [26] ont montré que la stimulation visuelle du chat correspondait à la mise en activation synchrone à $41 \mathrm{~Hz}$ de réseaux en boucles de neurones reliant de nombreuses zones du cerveau. Autrement dit, la mise en activation des ces réseaux est un phénomène qui s'exprime par « le chat voit».

Dans les réseaux humains en interaction, par exemple dans les réseaux financiers, les interactions réciproques créent aussi des boucles de rétroaction qui ont donné lieu à des modèles ad hoc. En revanche, les comportements humains au sein des groupes peuvent être beaucoup plus libres. La notion de libre arbitre ne peut être prise en compte que par des évaluations probabilistes. Les sociétés humaines, selon le nombre d'individus qui les composent et leur niveau de structuration, se tiennent entre ces deux pôles dont on détecte les attractions relatives dans l'examen de leurs comportements et on les inscrit dans des modèles qui en rendent compte. Je vais présenter sommairement des modèles qui se sont révélés très efficaces dans les branches des sciences sociales : démographie, géographie humaine, économie, finances et sociologie. Les problèmes rencontrés dans ces domaines débouchent sur des propositions d'ordre général pour assurer la cohérence des interprétations et leur scientificité.

\section{Démographie et géographie humaine}

Dans ce domaine, qui couvre un immense champ de recherche, des modèles très généraux se sont succédés. Courgeau [7] a fait une analyse de ce processus. D'abord, les données démographiques ont été interprétées globalement, sans référence aux comportements individuels, en considérant la Société comme un tout dotés de propriétés commandées par des variables.. Le modèle, par régression sur variables agrégées, était insuffisant et parfois aberrant, avec des résultats de probabilités supérieures à 1. Un modèle prenant en compte le déroulement des faits sociaux dans le temps évitait mes écueils du premier, mais devenait rapidement impraticable du fait du nombre de séquences qu'il devait prendre en compte. La prise en compte des données individuelles permit de surmonter les difficultés rencontrées jusque là, mais le fait global, en tant qu'émergence du cumul des comportements individuels n'était pas pris en compte. L'analyse multi-niveaux permit de prendre en compte les données individuelles en les insérant dans leur contexte sociétal, qui agit comme contrainte. Ce schéma s'apparente donc au schéma général de «Processus de Génération sous Contrainte » proposé par John Holland [16]. Je donne en Annexe 5 un article que m'a remis Daniel Courgeau [7] qui présente de manière très claire les résultats de l'application à un cas concret des trois « paradigmes » énoncés ci-dessus. On y voit combien le choix des paramètres et variables pertinents est crucial.

Deux domaines de la démographie ont donné lieu à des modèles intéressants. Les mouvements de populations, la formation éventuelle d'agrégats (dits " clusters ») ont été étudiés sur des modèles de graphes, dits modèles de Schelling, par Banos [1], avec un succès indéniable. 

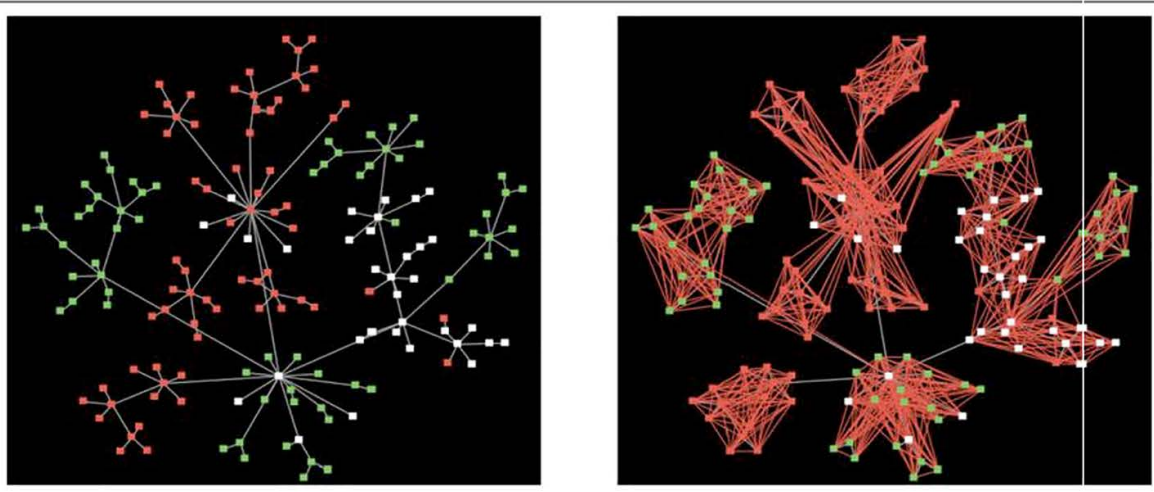

Figure 1. Les modélisations ci-dessus montrent que le déploiement de deux groupes de populations dépend du taux d'acceptabilité qu'elles manifestent entre elles. A gauche : bonne acceptabilité moyenne. A droite : refus d'interpénétration.

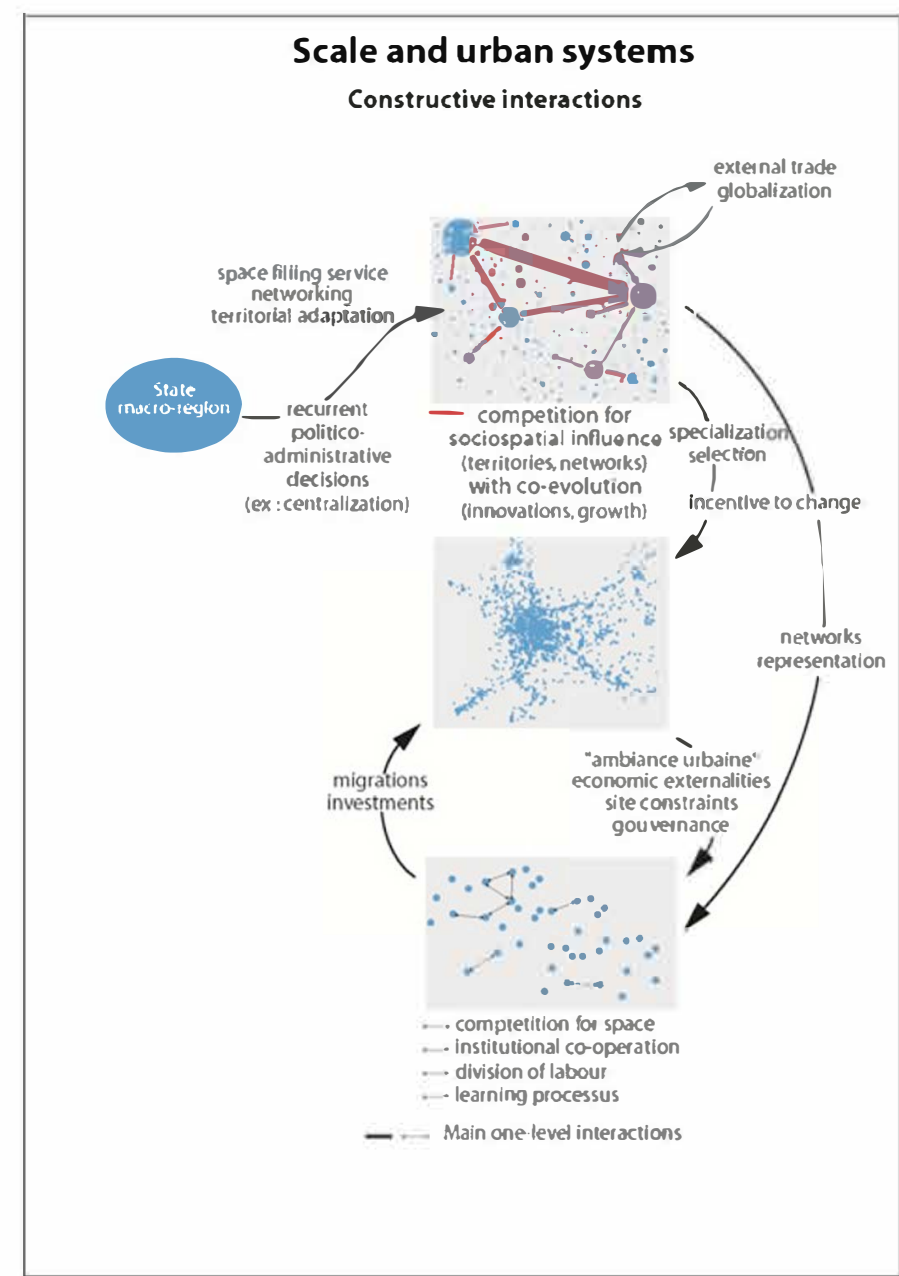

Figure 2. Le schéma ci-dessus nous montre l'enchaînement des relations que le modèle doit prendre en compte. 
Le développement urbain, en prenant en compte les interactions entre villes selon leurs spécificités géographiques et économiques, a été modélisé dans le Laboratoire de D. Pumain [25] (Université de Paris, PanthéonSorbonne), en mettant en œuvre des modèles à base d'agents. Ces modèles décrivent des comportements, alors que le modèle dit de Zipf [28], développé en modèle fractal par Mandelbrot [19], n'obtenait que des classements globaux. Toutefois, ces modèles ne peuvent pas prendre en compte ni les événements particuliers, ni les tendances lourdes héritées de l'histoire, et c'est par des simulations informatiques que le modèle atteint sa pleine efficacité.

Après cette revue des phénomènes concernant les groupes humains étudiés comme entités globales, $j$ 'en viens aux processus qui font interagir les individus, avec leurs capacités de conscience et d'anticipation. Ici interviennent les modèles en boucles de rétroaction

\section{Economie}

C'est à partir du $17^{\text {ème }}$ siècle que les réflexions sur les questions de gestion économique et financière ont commencé à recevoir des réponses. Elles ont été au départ des calculs probabilistes. Il s'agit de comprendre, d'une part, comment ça fonctionne, et d'autre part, comment ça va évoluer. La méthode est la méthode scientifique. On détecte les facteurs d'évolution, et on cherche, par un examen attentif, à créer entre eux des liens simples, ou à leur attribuer une évolution dans l'espace ou le temps. On en fait alors les variables dans des systèmes d'équations dont les résultats devront être confrontés à la réalité. Si les résultats sont favorables, on a un modèle et on peut tenter d'évaluer son évolution pour répondre à la deuxième question. Mais les paramètres et variables que l'on peut retenir sont si nombreux que les modèles peuvent avoir une ampleur démesurée (des centaines d'équations) et qu'il est très difficile de les hiérarchiser pour aller à l'essentiel. Toutefois, le domaine de l'économie reste dans une réalité faite de besoins et d'échange de matière ou d'énergie, et les événements y sont de ce fait moins soumis à des manipulations, des rumeurs et des actions perverses que ceux que l'on rencontre en finance.

\section{Comment ça va évoluer?}

Ici nous sommes dans l'évaluation des probabilités. Des tendances lourdes sont analysées, et elles ont une certaine continuité. Mais, dans tous les cas, la création et le contrôle du modèle doit donner lieu à un va et vient itératif qui est la méthode la plus efficace. Ainsi, le modèle a deux buts essentiels :

- d'une part, décrire de manière compréhensive une situation économique,

- d'autre part, de fournir des éléments d'appréciation permettant de prendre des décisions (politique économique, régulations, choix d'orientation).

En microéconomie, les interactions entre individus interviennent; les boucles de rétroaction doivent être prises en compte. Nous allons voir que les modèles développés dans la finance sont autrement plus complexes, et plus mathématisés qu'en économie. Cela tient sans doute au fait que de puissants intérêts personnels sont en jeu. Il y a là, évidemment, une question d'éthique, non seulement au plan individuel, mais aussi à celui des banques et des états, au plan global donc, car le volume total de la richesse n'est pas extensible, et que les manipulations financières tendent à accumuler la richesse chez les riches au détriment des pauvres.

\section{Les modélisations multi-fractales en finance}

Les successions d'événements en finance sont le fait d'acteurs qui attaquent la masse financière sous tous les angles possibles pour en tirer profit. Il est donc typiquement dans une situation analogue à celle des vagues qui attaquent les côtes. C'est un schéma d'interaction entre phases, qui appelle des modèles fractals. La multiplicité des acteurs a conduit à l'élaboration de modèles multi-fractals [12], c'est-à-dire à dimensions fractales multiples. Seuls ces modèles, en effet, permettent de répondre à une demande de schéma d'évolution ultra-rapide, dans un contexte où les transactions électroniques ont un temps propre de l'ordre de la milliseconde. (Calvet)

\section{Sociologie et sciences du management}

Depuis une dizaine d'année, la modélisation (et la simulation) multi-agents est perçue en sociologie comme une nouvelle méthode pouvant aider le chercheur à étudier de façon rigoureuse des ensembles complexes d'hypothèses qui portent sur la manière dont les acteurs agissent et interagissent. Encore faut-il que le comportement des entités virtuelles soit fondé sur des données statistiques et que les résultats macroscopiques du modèle soient confrontés à des données réelles spécifiques.

À la différence des théories physiques, une théorie sociale possède rarement une ou deux suppositions générales. Ainsi, la théorie du choix rationnel, due à Raymond Boudon [5], n'impose pas moins de six postulats disparates. Un modèle en science sociale est rarement le simple interprétant local et concret d'une théorie formelle unifiée et bien constituée. La complexification des modèles et l'essor des simulations sociales intégratives font que modèle et simulation deviennent assez proches et interagissent. 

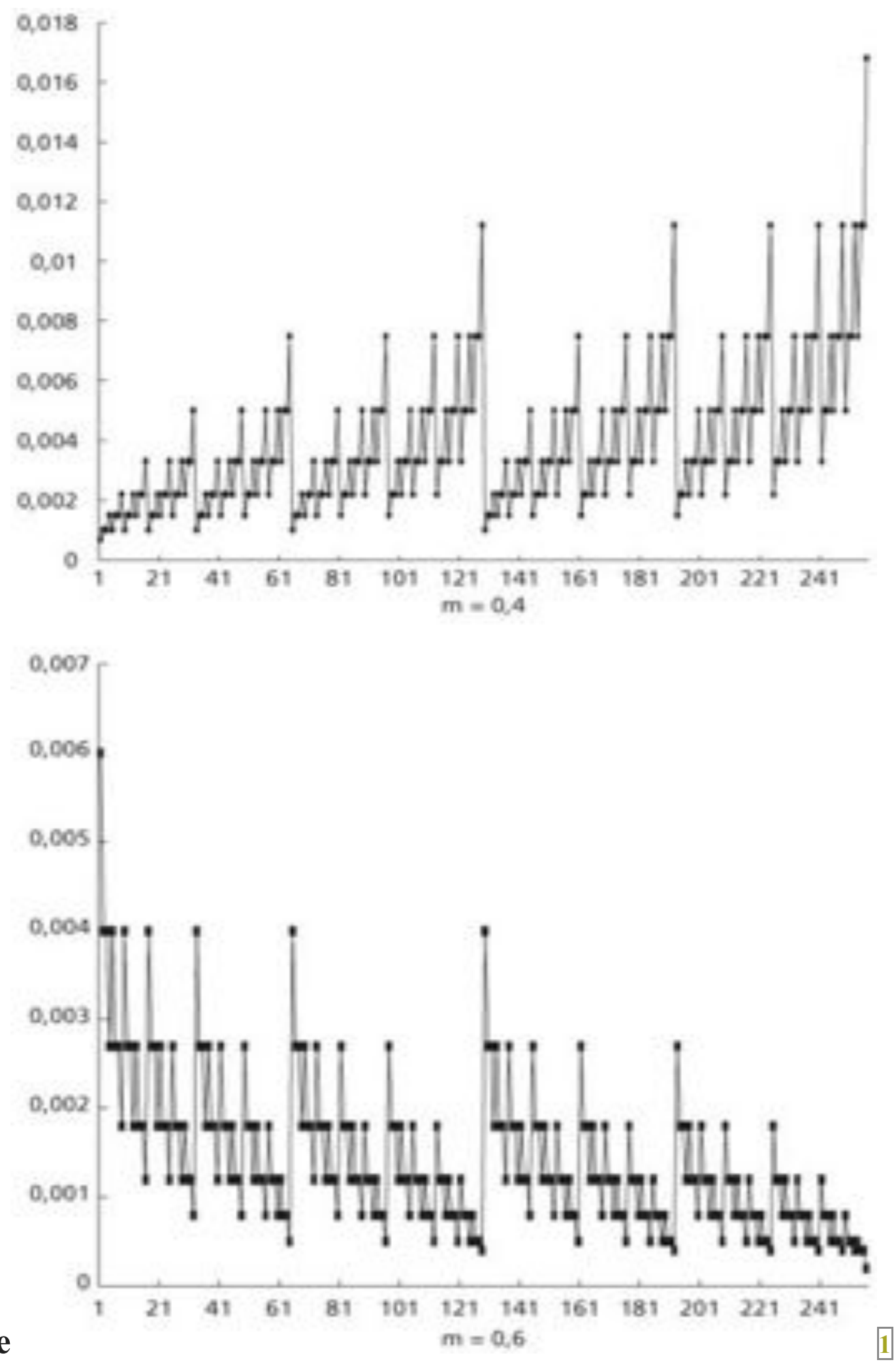

Dans les schémas ci-dessus, $m$ et m' sont les probabilités attribuées aux divisions successives du segment initial. Le tableau 1 est établi pour les valeurs 0,4 et 0,6 de $m$ et m', le tableau 2, pour $\mathrm{m}=0,6$ et $\mathrm{m}^{\prime}=0,4$. Tous ces travaux datent de moins de 30 ans. 
Les simulations tendent aujourd'hui à avoir la valeur épistémique d'un terrain empirique On ne doit pas pour autant renoncer à une modélisation compréhensive des phénomènes étudiés. Un recours aux données peut se faire par une approche ethnographique, qui est descriptive, voire explicative, une approche quantitative, qui permet d'établir des relations entre variables, et par l'expérimentation. qui décrit la variation des comportements en fonction des conditions de l'expérience.

Nous avons, au cours de ce colloque, un exemple limpide d'un tel modèle, présenté par Didier Cuménal, sur le thème : "obsolescence des organisations ». Ce modèle réunit dans ses prémisses les diverses approches énoncées ci-dessus, et il met en oeuvre le principe des « variables les plus pertinentes, et de leur test (équations différentielles aux dérivées partielles).

Les comportements individuels sont variés, mais ils subissent tous les contraintes de l'environnement social, c'est à dire les règlements et les lois, les usages, ce qui constitue un ensemble qui n'a pas la rigueur des lois de la physique, mais qui permet de rendre compte des données empirique. Notons que ces phénomènes peuvent répondre à la notion de «causalité descendante », puisqu'il s'agit bien de l'action de propriétés du « tout 》 (les institutions de la société) sur les parties (les individus). Je note malgré tout que ce n'est pas le code de la route qui agit sur les conducteurs, mais bien la peur du gendarme ou l'adhésion du conducteur, résultat de son éducation, qui font que le code de la route est généralement respecté, et non quelque force émanant du « CODE ». Je tiens à signaler ce point pour attirer l'attention sur le fait que, dans tous les autres cas où l'on rencontre l'hypothèse de « causalité descendante (Top-Down Causation), les auteurs qui avancent cette notion seraient bien incapables de décrire comment le tout agit sur les parties, autrement que par les interactions des parties elles-mêmes.

Les comportements sociaux ont été étudiés depuis l'antiquité, mais c'est au début du $18^{\text {ìme }}$ siècle que la sociologie est née. Lorsque Darwin a produit sa théorie de l'évolution, un courant philosophique né en Angleterre a érigé en principe la lutte pour la vie. A la fin de sa vie, Charles Darwin [10], très affecté par cette interprétation, a exposé les conditions qui conduisent à l'apparition de comportements non égoïstes chez les animaux supérieurs et chez l'homme. L'altruisme y apparait comme un comportement « sélectionné par l'évolution ». En effet, le rapport intime de l'individu à son entourage dans les groupes sociaux entraine des comportements affectifs qui sont à l'opposé de la notion de lutte individuelle pour la vie. Chez les êtres humains raisonnants, nous voyons apparaître ce que Cresson [8] appelle « l'intérêt bien entendu », qu'il invoque au nom de Kant. Les problèmes posés par la gestion des groupes humains, par le comportement équilibré des individus dans leur groupe, familial, professionnel, associatif, d'une manière plus générale les problèmes de gouvernance se résolvent par la nécessité de la coopération, comme solution la plus avantageuse. Cette notion est érigée en principe énoncé par Pierre Bricage [6] : celui des «avantages et inconvénients mutuellement acceptés et partagés ». Ce sera le dernier point de cette revue des dynamiques qui vont de l'inerte atomique au vivant humaniste.

\section{CONCLUSION}

Grâce à la puissance et à la capacité de mémoire des ordinateurs, rien ne s'oppose aujourd'hui à un projet d'élucidation de tout phénomène naturel. Il faut seulement adapter les outils classiques de la physique, en inventer d'autres aussi, mais en fondant toujours nos recherches sur les capacités d'induction de notre rationalité. Les représentations que nous nous formons du réel acquièrent un statut scientifique par la rigueur des formulations mathématiques ou des modèles qui en sont l'expression la plus générale.

Mais, à partir de l'explosion biologique, la complexité impose des modèles non-linéaires que nos mathématiques actuelles commencent seulement à aborder. Les processus de développement sous les contraintes environnementales, dits "émergences ", sont aujourd'hui élucidés de proche en proche. La complexité s'accroit considérablement chez les êtres vivants dotés d'un système nerveux central qui leur confère une capacité de réaction à l'environnement qui devient, au cours de l'évolution, une capacité de représentation consciente de l'environnement (chimique et (tactile d'abord, puis visuel). Enfin, chez les animaux supérieurs, apparait la capacité d'anticipation, que l'homme développe largement grâce à une construction mentale de la durée. En outre, chez l'homme, l'élément essentiel est la relation avec ses semblables avec l'apparition du langage. L'intersubjectivité qui en résulte peut être exploitée en tant que contrainte qui s'impose aux individus. Dans ces conditions, certains individus peuvent jouer un rôle décisif sur l'évolution de leur groupe humain, voire de l'humanité. L'histoire nous donne à foison des exemples d'enjeux de pouvoir qui sont autant de perversions militaires, politiques et religieuses imputables à des individus ou à de petits groupes. La finance est aussi un lieu de telles perversions dues à la cupidité des acteurs, tant au plan individuel qu'à celui des entreprises et jusqu'au niveau des nations. 
Pourtant, dans une société d'hommes libres, les attitudes fondées sur l'intérêt bien entendu devraient prévaloir. Mais la période de bouleversement démographique et d'inégalités profondes que nous vivons n'est pas favorable à l'émergence de l'adoption mutuelle, entre partenaires, des "avantages et inconvénients réciproques et partagés » (Pierre Bricage [6]). Contre le règne de la loi du plus fort, qui institue la compétition dès l'enfance, certains idéalistes persistent à croire en l'homme : André Gide disait, dans un discours à la jeunesse en 1946 : « Je crois à la vertu du petit nombre. Le monde sera sauvé par quelques-uns. ».

\section{RÉFÉRENCES}

[1] D. Badariotti, A. Banos, D. Moreno, "Conception d'un automate cellulaire non stationnaire à base de graphe pour modéliser la structure spatiale urbaine : le modèle Remus." Cybergeo, 2006, article 403, http://www.cybergeo.eu/index10993.html

[2] A. Baldassari, In "La Percolation en gradient : des fronts de diffusion aux fronts de mer.", Colloque " Emergences », AEIS, Paris, 2008.

[3] R. Balian, "Du microscopique au macroscopique." Ellipses, Paris, 1990.

[4] M. Ben Amar, "Morphogenèses et embryogenèses." conférence AEIS, Paris, Février 2013, http://www.science-inter.com

[5] R. Boudon, "Les méthodes en sociologie." Que sais-je ?, PUF, Paris, 2002.

[6] P. Bricage, " Systèmes vivants : agoantagonisme inter- et intra- niveaux.", AFSCET-CNAM-AEIS, UES-EUS, 2014, http://web.univ-pau.fr/ bricage/files/bricage_s02-5c-paper.pdf

[7] D. Courgeau, "Changement de paradigme en démographie.“, Population, vol 51, n³.

[8] A. Cresson, "Le problème moral et les philosophes.", Collection scientifique Armand Colin, Paris, 1935.

[9] P. Curie, "Sur la symétrie dans les phénomènes physiques.“, Journal de physique (repris par EDP Science), 1894.

[10] Ch. Darwin, "L'expression des émotions chez l'homme et les animaux.“, John Murray, 1877.

[11]E. di Mauro, “À propos de la génération moléculaire spontanée.“, colloque AEIS, Systèmes solaires et planétaires; conditions d'apparition de la vie, AEIS-EDP, 2014, à paraître.

[12] J. Fillol, "Modélisation multifractale du taux de change dollar/euro." Economie internationale 2005/4 (n 104), La Documentation Française, Paris.

[13] M. Filoche, "Efficacité physiologique des arbres fractals : le cas du poumon humain." In Irruption des géométries fractales dans les sciences », AEIS, 2005

[14] R. Franck, "The explanatory power of the Models." Kluwer Methodos Series, 2002.

[15] M. Gell Mann, "Le quark et le jaguar." Champs, Flammarion, 1997.

[16] J. Holland, "Emergence. From chaos to order.“"New York, Basic books. 1998.

[17] Lambert et Rezsöhazy, "Comment les pattes viennent aux serpents.“, Flammarion, 2007.

[18] G. Losa, "La géométrie fractale dans la compréhension de la complexité biologique." In « Emergences », ed. AEIS, Paris

[19] B. Mandelbrot, "Les objets fractals.", Champs Flammarion, 1984.

[20] F. Molino, "Biologie systémique et émergence : le système neuroendocrinal, une étude de cas." Colloque «Emergences », AEIS, 2008, à paraître.

[21] J. Monod, "Le hasard et la nécessité.“, Le Seuil, Paris, 1970.

[22] La loi de Moore, formulée en 1965, prévoyait le doublement du nombre de transistors, et de la puissance des ordinateurs tous les 18 mois.

[23] On arrive aujourd'hui à la limite (dimensionnelle) des transistors, mais surtout, à des problèmes liés à l'accumulation des déchets qui est de plus en plus alarmante. Informatique et développement durable,

[24] D. Noble, "La musique de la vie", Le Seuil, Science ouverte, 2007.

[25] D. Pumain, "Alternative explanations of hierarchical differentiation in urban systems.", In Pumain D. (ed.) Hierarchy in natural and social sciences, Springer, Methodos series 3, 169-222.

[26] W. Singer, "Une coordination temporelle à grande échelle comme condition préalable à l'expérience consciente.", in Biologie et conscience, ed. AEIS, 2002.

[27] B. Walliser, "Systèmes et modèles.", Le Seuil, Paris, 1977.

[28] Zipf Voir Mandelbrot [19]

\section{ANNEXES}

Annexe 1. Du microscopique au macroscopique [3]. Du probabilisme au déterminisme : une question d'échelle. Annexe 2. Les émergences, les morphogenèses, une conclusion épistémologique.

Annexe 3. Modélisations des structures complexes dans la nature. Leurs développements et leurs emboîtements.

Annexe 4. Développement des performances cognitives au cours de l'évolution.

Annexe 5. Les migrations en Norvège des agriculteurs (Daniel Courgeau) [7]. 


\section{ANNEXE 1}

\section{Du microscopique au macroscopique . Du probabilisme au déterminisme : une question d'échelle.}

Monod* et Jacob* ont instauré le déterminisme génétique selon l'idée générale, qui relève du bon sens, que la rigueur des embryogenèses provient d'une programmation rigoureuse. Beaucoup de biologistes sont d'accord pour accepter une forme de téléonomie, selon le vocable utilisé par Monod* pour éviter de parler de prédestination.

Mais si l'on pose simplement la question de savoir comment se forment les ARN, les enzymes, les protéines, on doit aller au niveau spatial et temporel des molécules pour appréhender les phénomènes. On ne peut, au plan macroscopique, que constater que l'ADN est une grande structure chimique qui produit toutes sortes de molécules sans subir elle-même aucune modification, l'ADN est, au sens strict du terme, un catalyseur.

Que l'on dise, comme Denis Noble, que c'est une banque de données, ou comme la plupart des biologistes, que l'ADN « exprime une information », ces termes peuvent sous-tendre deux conceptions opposées : soit une téléologie, soit le principe darwinien selon lequel les assemblages moléculaires favorables ont subsisté au détriment des autres.

Quelles que soient les interprétations, il faut en venir au niveau moléculaire pour prendre la mesure spatiale et temporelle des phénomènes. Les molécules présentes dans le milieu cellulaire forment, par catalyse sur l'ADN, les ARN et autres grandes molécules qui structurent la matière vivante. Dans leur parcours erratique, les molécules qui rencontrent l'ADN, peuvent s'y fixer, retenues par les forces de Van der Waals. Des molécules, ainsi fixées, s'y associent et forment une grosse molécule que les forces de Van der Waals n'empêcheront pas de se détacher de l'ADN et iront, au hasard des rencontres, provoquer un métabolisme. Le phénomène est identique à celui qu'on montre aux enfants de douze ans en faisant la synthèse catalytique de l'eau sur de la mousse de platine.

Ainsi, les forces de Van der Waals sont largement responsables des phénomènes biologiques. Encore faut-il avoir présent à l'esprit, pour comprendre que ces phénomènes ne sont pas guidés par quelque force mystérieuse, qu'ils ne sont pas du tout instantanés à leur échelle de temps. Il faut prendre en compte le fait que la fréquence des mouvements moléculaires dans le milieu biologique est de l'ordre de la nanoseconde, voire de la femtoseconde. A l'échelle de la femtoseconde, la seconde représente l'écart relatif qu'il y a entre une seconde et 33.000 ans ! Il se passe beaucoup, de choses, dans notre échelle de temps, en 33.000 ans :

Ainsi, toute cette accumulation de phénomènes microscopiques, qui finissent par des moyennes très stables et des variances très faibles malgré leur caractère stochastique, nous apparaît dans un déterminisme strict : le fameux déterminisme génétique.

A l'échelle de temps des molécules, les événements sont innombrables, leurs résultats peuvent n'être que rarement durables, c'est-à-dire stables, mais ils sont si nombreux, et se déroulent dans des délais tellement infimes qu'ils nous apparaissent comme instantanés, continus et strictement déterminés.

La complexité biologique est alors le résultat stable d'innombrables essais et erreurs parmi lesquels quelques succès subsistent. Mais ces nombres sont tellement gigantesques que le processus de formation du vivant se déroule, et que, même après des catastrophes écologiques, la vie a repris son cours, comme la paléontologie nous le montre. 
Annexe 2

Les émergences, les morphogenèses, une conclusion épistémologique.

En biologie, on utilise souvent le terme d'émergence pour qualifier des processus qui produisent des structures dotées de propriétés «nouvelles ».

Beaucoup d'ambiguïtés ont accompagné les recherches tout au long du 20ème siècle.

- Après des décennies de confusion où les phénomènes de transition de phase, au sens le plus général, étaient qualifiés d'émergences, et considérés comme des «novations absolues » et classés dans l'ordre du mystère. Tous les mystères, progressivement s'effritent et on voit poindre des théories générales susceptibles de prendre en charge toutes les morphogenèses et les embryogenèses. L'idée que la connaissance des propriétés des éléments et celle de leurs interactions, entre eux et avec leur environnement, est nécessaire et suffisante pour connaître toutes les propriétés de l'ensemble qu'ils forment, devient vérité d'expérience. Les travaux de John Holland*, co-fondateur avec Murray Gell Mann* du Santa Fe Institute (for complexity studies), le conduisent à formuler sa théorie des « Processus de génération sous contrainte » (Constraint Generative Procedure CGP ). Cette théorie s'appuie sur la similitude étonnante de deux modèles mis à l'épreuve de l'expérience : le modèle du joueur de dames de Salomon, et celui des réseaux neuronaux du même Holland. Je me bornerai ici à donner, sur les pas de Holland, la définition suivante du mot émergence : « processus de transition entre deux niveaux d'observation ».

Cette définition se fonde

- Sur le fait que le phénomène émergent est un processus, mettant en jeu les divers éléments du système étudié, entre eux et avec leur environnement, dans un déroulement dont l'aboutissement est la création d'un niveau d'organisation englobant les éléments du système dans son niveau d'organisation

- - sur l'acceptation épistémologique que le scientifique, dans son effort de représentation des phénomènes naturels, est responsable du choix des variables et paramètres pertinents, du modèle de sa représentation, et du choix des niveaux où il décide d'appliquer son modèle.

- On est ici, évidemment à l'opposé d'une interprétation ontologique.

Vers des théories ou des modèles généraux pour les morphogenèses ?

L'idée générale qui tend à prévaloir aujourd'hui est la suivante :

Le développement cellulaire dépend essentiellement des qualités nutritionnelles du milieu cellulaire. Abondant, il provoque le développement par mitose des cellules : insuffisant, il provoque leur mort (apoptose).

Mais si ce développement n'est pas guidé, il est anarchique : c'est le cancer. En revanche, soumises aux contraintes de l'environnement, les cellules de développent avec une extraordinaire plasticité qui est à l'origine de l'immense variété des formes naturelles.

L'étude des développements qui prend en compte les contraintes de l'environnement est la voie royale pour l'élucidation des morphogenèses 
Les chercheurs constatent que les mesures et les calculs qu'ils doivent effectuer dépendent largement des performances des outils informatiques dont ils disposent. Depuis quarante ans, la loi de Moore, qui décrit le taux exponentiel de croissance de capacités et des vitesses des ordinateurs leur permet de prévoir à quel moment ils pourront atteindre tel ou tel résultat. Il faut noter que cet accroissement exponentiel va sans doute faire une pause, car on arrive à la limite dimensionnelle des transistors. Quoi qu' on puisse inventer, il y aura une limite à cet accroissement. La création humaine est liée à une longue maturation des idées, mais cette maturation n'est pas programmable. En revanche, elle dépend du développement technique. La nature a mis des centaines de millions d'années pour produire des oiseaux. Arrivé à un certain niveau technologique, l'homme a, en un instant par rapport à ces âges géologiques, construit des avions et il en sillonne le ciel. (Léonard de Vinci avait conçu des aéronefs, mais il était trop en avance sur les techniques de son temps pour pouvoir les réaliser).

Ceci a une grande portée épistémologique : La nature est faite de matériaux qui se sont constitués au cours de la formation de l'univers. Ces matériaux se sont rassemblés selon les concours de circonstances. Ces assemblages ont été dotés de propriétés qui les ont conduits à produire des fonctionnalités. C'est le schéma darwinien qui va des causes matérielles d'Aristote aux causes formelles, puis aux causes efficientes. La nature, dans ce schéma, ne connait pas de causes finales.

En revanche, l'homme, dans son intention, bâtit un projet, définit un but : par exemple, transporter par la voie aérienne. Il en déduit les capacités nécessaires à son projet (causes efficientes), puis imagine les structures nécessaires (causes formelles) et enfin définit les matériaux de sa construction.

C'est le schéma aristotélicien inverse du schéma darwinien de la nature.

Toute interprétation des phénomènes naturels selon le schéma qui part des causes finales est un anthropomorphisme. 


\section{ANNEXE3}

\section{Modélisations des structures complexes dans la nature. Leurs développements et leurs emboîtements.}

Les premières recherches ont concerné la dynamique des fluides. , au cours du $19^{\text {ème }}$ siècle, les travaux des analystes, théorème de Navier-Stokes). A la fin du $19^{\text {ème }}$ siècle apparut en Angleterre le courant émergentiste, qui, faute de moyens d'investigation, laissa planer beaucoup de mystère dans les phénomènes dits émergents. Etait considéré comme émergents un phénomène dont on ne pouvait pas comprendre l'avènement.

Il faudra attendre l'arrivée des ordinateurs pour aborder avec succès la résolution des phénomènes complexes.

Entre temps, des travaux avaient largement déblayé le terrain.

D'abord, des développements considérables dans la mécanique des fluides et des matières molles, où la plasticité apparaît comme une caractéristique majeure. ( Lambert* et Rezsöhazy*)

Je donne ci-aprés un exemple de modélisation mathématique d'une croissance végétale due à Martine Ben-Ammar*.

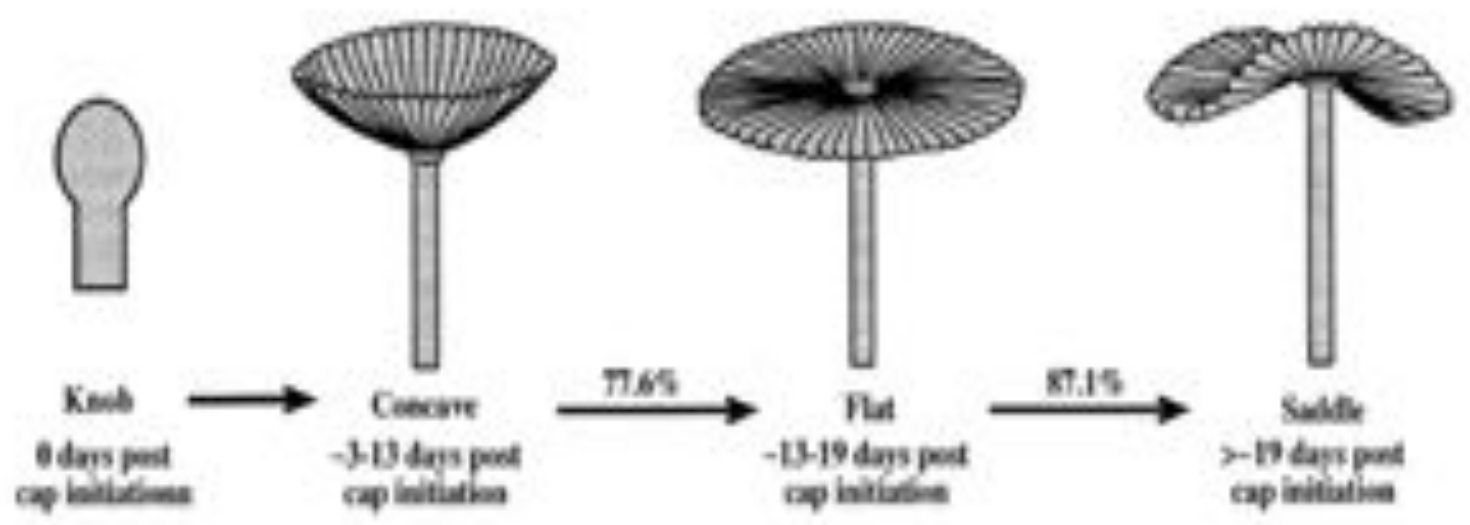



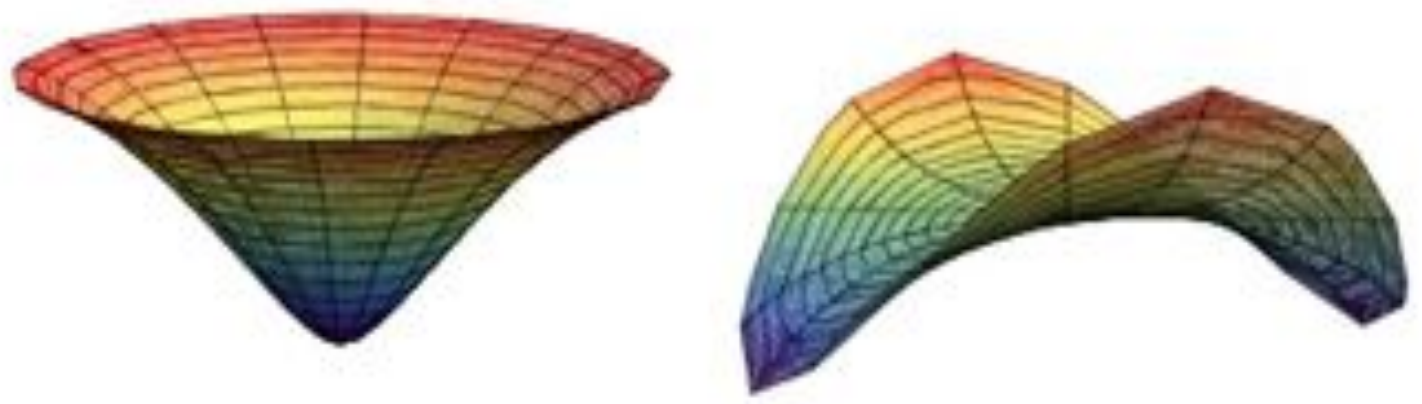

Le schéma ci-dessus montre les stades successifs du développement d'un champignon, et, en regard, deux surfaces correspondant à deux stades de la fonction d'évolution rendant compte de cette morphogenèse.

Puis, à partir de 1973, ce fut la révolution des fractales, due à Benoît Mandelbrot. Les bases mathématiques avaient été jetées tout au long du20ème siècle, mais Mandelbrot eut le génie d'appliquer les géométrisations fractales à tous les phénomènes ou des phases interagissent : fronts de toutes sortes, interdiffusions, corrosion, électrolyse, , ravinements (fluviaux, glaciaires), mais aussi tous phénomènes interprétables en termes d'interpénétrations entre phases, , ainsi que les structures en branchements successifs pour atteindre les contacts ultimes dans la plus grande efficacité (poumon, rein)..

Je donne ci-après un schéma d'un front de diffusion (gravure à l'acide ). Au fur et à mesure, l'acide s'épuise à attaquer le métal et le front d'interface, linéaire au départ, devient de plus en plus fractal. (étude due à Baldassari*).

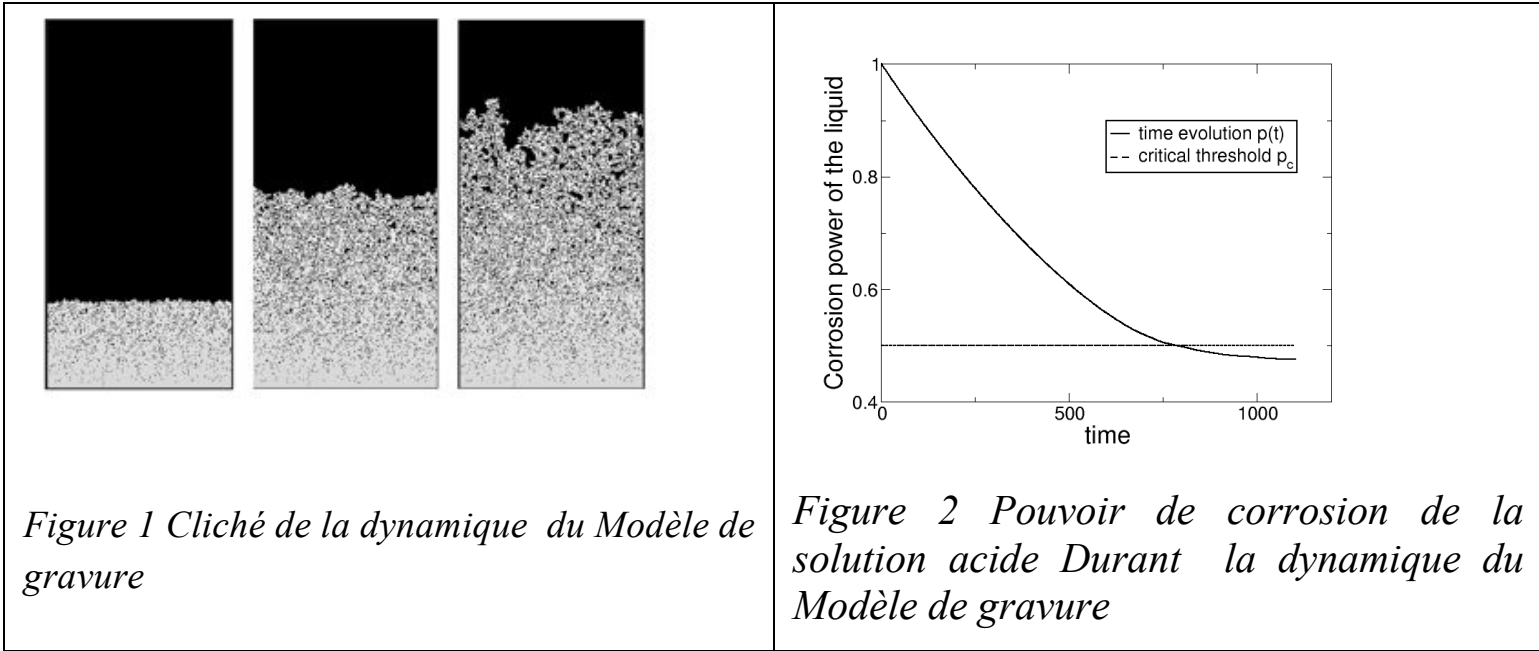

Des recherches avec formulations fractales très fructueuses ont été accomplies dans tous les domaines de la biologie où se manifestent des affrontements de populations cellulaires (cancers), et là où les relations entre tissus nécessitent une interaction microscopique (par 
exemple poumon, rein). Les travaux de Filoche* sur la structure fractale du poumon sont le meilleur exemple de ces développements. Lorsque des tissus sont en affrontement, par exemple dans le cas du développement d'une tumeur cancéreuse, le front d'interaction a une structure fractale dont l'imagerie rend compte, et elle permet un diagnostic précoce. (Losa*).

La modélisation des développements biologiques et des phénomènes de la physiologie est puissamment fondée sur l'induction minutieuse et les tests de variables pertinentes ( principe des tests de variables par équations différentielles aux dérivées partielles.

Denis Noble* s'est illustré par ses travaux sur le cœur humain, -description et explication complète des pulsations cardiaques --, et le Laboratoire de Génomique fonctionnelle de l'Université de Montpellier a décrypté totalement le fonctionnement de l'hypophyse du rat. Ce résultat, dû à François Molino*, a pu être obtenu grâce à la capacité des ordinateurs de prendre en charge les fonctions à partir du niveau de la cellule et des molécules que les cellules produisent. (cette glande possède environ 600.000 cellules).

Deux images 3D des états non excité et excité de l'hypophyse du rat (Molino*).
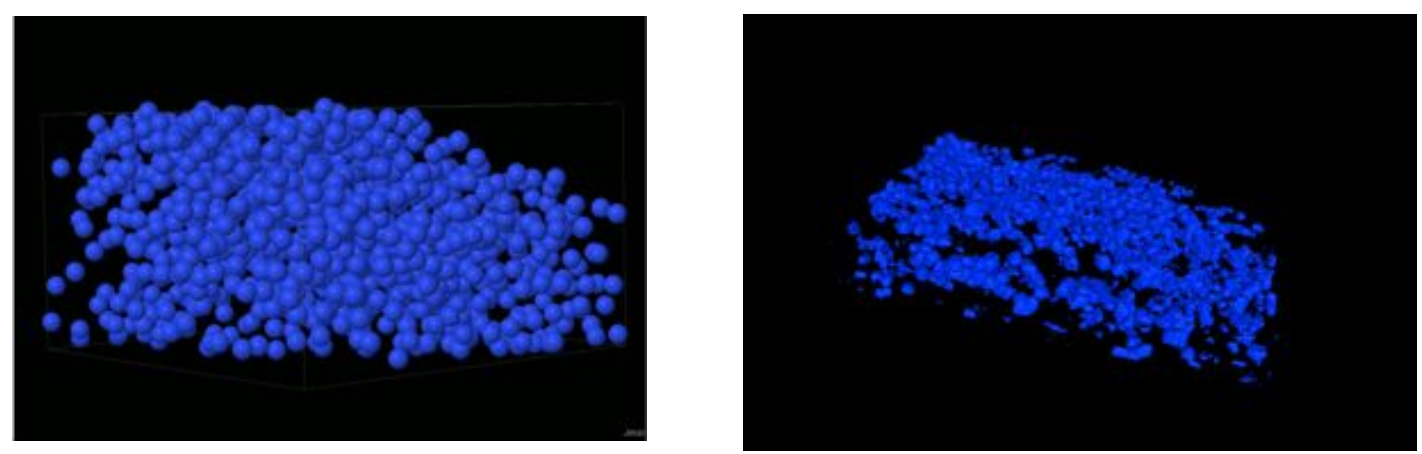

La représentation $3 D$ de l'hypophyse du rat montre la réorganisation des enchaînements cellulaires lors de l' excitation électrique synchrone des cellules (étude et clichés de Molino*, Laboratoire de génomique fonctionnelle, Université de Montpellier). Cette étude analyse le fonctionnement de l'hypophyse du rat à partir des molécules, par niveaux successifs d'intégrations, jusqu'aux relations entre organes. Etude intégrative, typiquement " bottom-Up, , par tests de variables pertinentes, c'est-à-dire test sur équations différentielles aux dérivées partielles).

Les modèles algébriques ont toujours une valeur primordiale, mais des modèles fondés sur des fonctions d'itération, largement développés par von Neumann et surtout, - encore lui -, Mandelvrot, ont de plus en plus d'intérêt, car ils s'adaptent parfaitement au travail séquentiel des ordinateurs. C'est d'ailleurs la raison du succès des automates cellulaires. Cependant, dans la plupart des applications, strictement déterministes, ces modèles sont peu adaptés à la mouvance et à la plasticité des phénomènes du vivant. 
Toutefois, des automates cellulaires probabilistes ( Kupiec*), ont une valeur heuristique. De même, j'ai présenté dans le texte les modèles d'Arnaud Banos*, modèles automates en graphes, issus des modèles de Schellig.

Les modèles en niveaux successifs de structurations sont particulièrement adaptés aux stades successifs des embryogenèses, et par suite aux structurations complexes des êtres vivants? Mais ils trouvent, comme je l'ai évoqué dans le texte, de nombreuses applications en sciences sociales.

Enfin, les modèles en boucles de rétroaction s'imposent dès que les agents interagissent. Ils sont aussi les modèles privilégiés des processus du système nerveux central, car ils correspondent à une réalité expérimentale. (Singer*). 


\section{ANNEXE 4}

\section{Développement des performances cognitives au cours de l'évolution.}

On entend parfois dire « les cellules communiquent entre elles ». Ceux qui le disent pourraient-ils expliciter le genre de communication dont il s'agit ? Si les membranes des cellules sont sensibles à un taux critique de concentration de certaines molécules, et qu'elles réagissent en conséquence,, peut-on assimiler sans grossièreté ce mécanisme chimique à une communication ? et trouverait-on chez les cellules des phénomènes d'envois « délibérés » de molécules, ou de « signaux », (mais de quel type ?) ?, manifestant en quelque sorte une « intention » de la cellule?

Attention aux anthropomorphismes, tellement séduisants !

Pour qu'il y ait information, il faut qu'il y ait un émetteur, et un récepteur, prêt à recevoir l'information en tant que donnée à intégrer dans un réseau qui provoque une réaction, résultat d'un traitement. Si bien que,pour parler d'information autrement que par une métaphore d'une poétique approximative, il faut que le système qui utilise un tel moyen soit équipé d'un système de traitement de quelque signal que ce soit.

L'évolution a sélectionné les réseaux neuronaux d'un système nerveux pour accomplir cette fonction.Mais ce système nerveux n'a de capacité informationnelle que s'il traite l'information, c'est-à-dire s'il forme, grâce aux perceptions, une représentation même rudimentaire de l'émetteur. C'est l'existence d'un système nerveux central qui permet cette fonctionnalité de haut niveau.

Les êtres vivants multicellulaires, équipés d'un système nerveux central, forment des représentations de leur environnement. Ils sont, de ce fait, beaucoup mieux en mesure de survivre, au niveau des espèces, aux événements qui leur sont défavorables ; ils se reconnaissent entre membres d'une même espèce, et ils acquièrent des comportements individuels et collectifs.

Les insectes sont strictement déterminés par leurs instincts, Leur niveau d'individuation est minimal ; ils agissent sans se préoccuper de leur sort, et d'après les naturalistes, la plupart meurent par des accidents qu'ils n'ont pas la capacité de prévoir, encore moins d'éviter.. Il n’y a donc chez eux, ni égoïsme, ni coopération.Il n'y a pas de compétition ni de dévouement. Abordons les vertébrés :

Les bancs de poissons ne présentent ni structure familiale, ni sociale.

Les oiseaux élèvent leurs petits, ils leur apprennent à chanter et se rassemblent pour des migrations très complexes.

Les mammifères supérieurs vivent en famille, du moins pour la durée de l'élevage des petits, ils se forment en hordes, , ils se défendent collectivement des prédateurs.

Chez les singes supérieurs proches de l'homme, des pratiques d'apprentissage, de confection et d'appropriation d'outils apparaissent, des pratiques de type cultuel des formes de solidarité, de la compassion ou de la méfiance se manifestent Chez l'homme, ces comportements sont encore plus complexes, du fait de l'apparition du langage conceptuel, symbolique.

Comme le langage est imposé au nouveau-né par son entourage, cet entourage lui impose aussi un mode de fonctionnement cérébral, si bien que le langage, la pensée 
individuelle, la " pensée émanant du groupe », que j'appellerai pensée collective, sont indissociables. Et c'est par cette intersubjectivité que la complexité humaine explose.

En outre, à l'instar sans doute des animaux supérieurs, l'homme mémorise la succession des événements en créant la durée, et en la projetant dans l'avenir. Si bien qu'il est capable d'anticiper ses propres actions, mais aussi les comportements de ses semblables, et de son environnement.

J'ai relaté dans le texte les travaux qui ont conduit à l'élaboration, à ce niveau de complexité, de modèles en boucles de rétroactions. 


\section{ANNEXE 5}

Je donne ci-après une étude de Daniel Courgeau qui analyse les résultats des paradigmes successifs qui ont prévalu en démographie. Ces résultats antinomiques montrent la nécessité cruciale du choix des paramètres et variables pertinents.

\section{Les migrations en Norvège des agriculteurs}

\footnotetext{
${ }^{1}$ Nous remercions ici les services statistiques norvégiens qui nous ont permis d'avoir accès à des fichiers créés à partir de ces données et des recensements de population par Kjetil Sørlie et Øjsten Kravdal
}

\section{Approche agrégée}

Je considère les migrations entre régions norvégiennes observées par le registre de population, données qui furent centralisées et informatisées en $1964^{1}$. L'histoire résidentielle complète de chacun de ces individus est connue à partir de 1964, mais le souci des services statistiques norvégiens de préserver l'anonymat des individus, m'a conduit à travailler au niveau régional, la Norvège étant divisée en 19 régions. J'ai éliminé les individus ayant eu au moins un séjour hors de Norvège. 


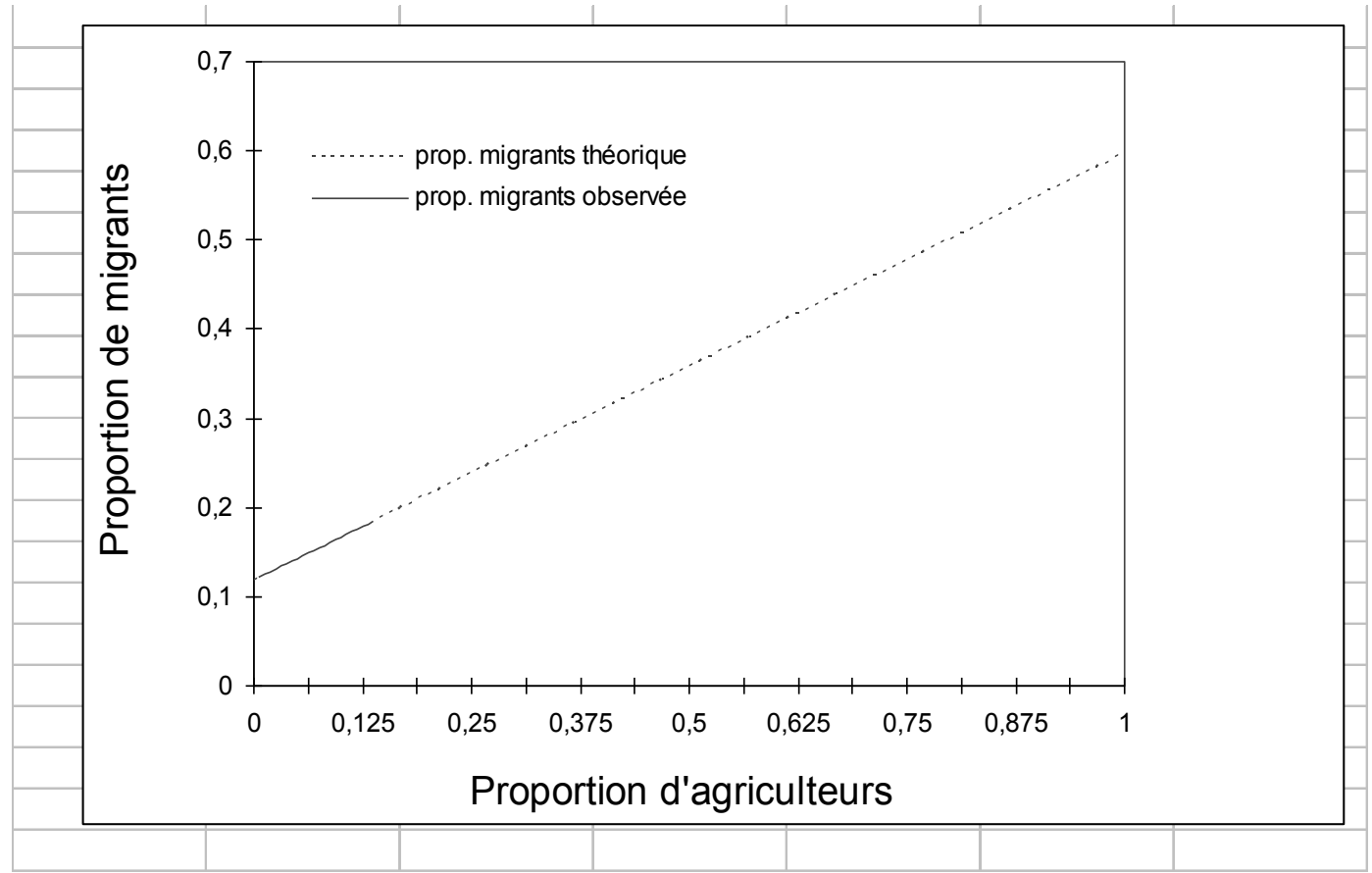

J'observe ici tous les hommes nés en 1948, résidant en Norvège en 1991, soit un total de 28462 individus. Je considère les changements de région effectués sur une courte période de deux ans suivant le recensement de 1970 et considère ici les probabilités de migrer des agriculteurs comparés aux autres membres de la population, en travaillant sur les données agrégées.

A partir de ces données il est possible de calculer une régression linéaire, pondérée par les populations régionales, entre les taux de migration $y(r)$ et les proportions d'agriculteurs $x$ de chaque région. Cette estimation pondérée fournit la droite de la figure 1. 
Figure 1. Taux de migration en fonction de la proportion d'agriculteurs

Les probabilités estimées montrent qu'il y a une liaison positive, significative, entre le taux de migration et la proportion d'agriculteurs de chaque région. La part de variance expliquée est de 0,24 et est significativement différente de zéro au seuil de $5 \%$. On peut donc conclure que les agriculteurs en Norvège ont une probabilité près de six fois plus forte que les autres professions de migrer, si les hypothèses du modèle sont vérifiées. Ce résultat peut paraître surprenant étant donné le coût tant financier que personnel d'un changement de région pour cette profession. Nous verrons également plus loin que certaines des hypothèses à la base de cette estimation peuvent être incorrectes.

\section{Approche biographique}

On dispose maintenant des données individuelles des membres de la génération née en 1948 et observée au cours de la période de deux ans suivant le recensement de 1970. L'ajustement d'un modèle logit à ces données conduit à la

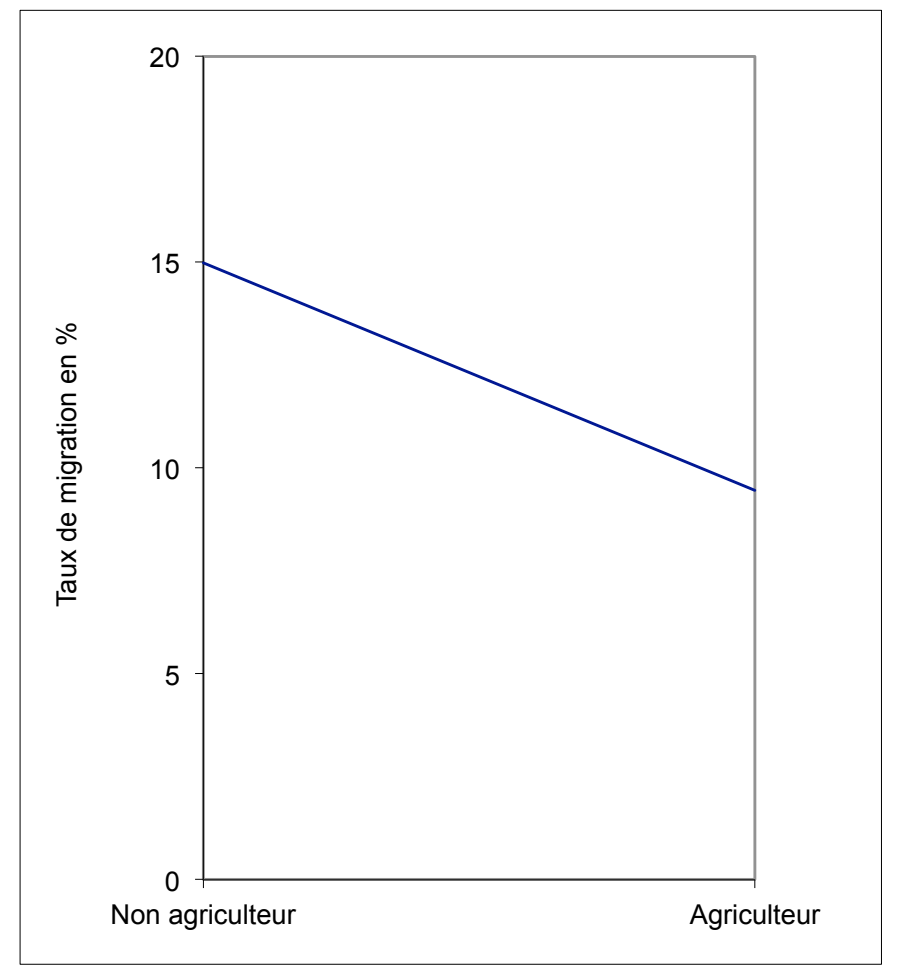

figure 2 .

Figure 2. Taux de migration des agriculteurs et des 
On voit donc que, contrairement à ce que l'on avait montré avec les données agrégées la probabilité de migrer des agriculteurs est plus d'un tiers inférieure à celle des autres professions et, comme je l'avais indiqué cette estimation est plus conforme à ce que l'on attendait.

\section{Approche multiniveaux}

Faisons maintenant intervenir simultanément le fait que l'individu soit agriculteur et les proportions d'agriculteurs présents dans chaque région.

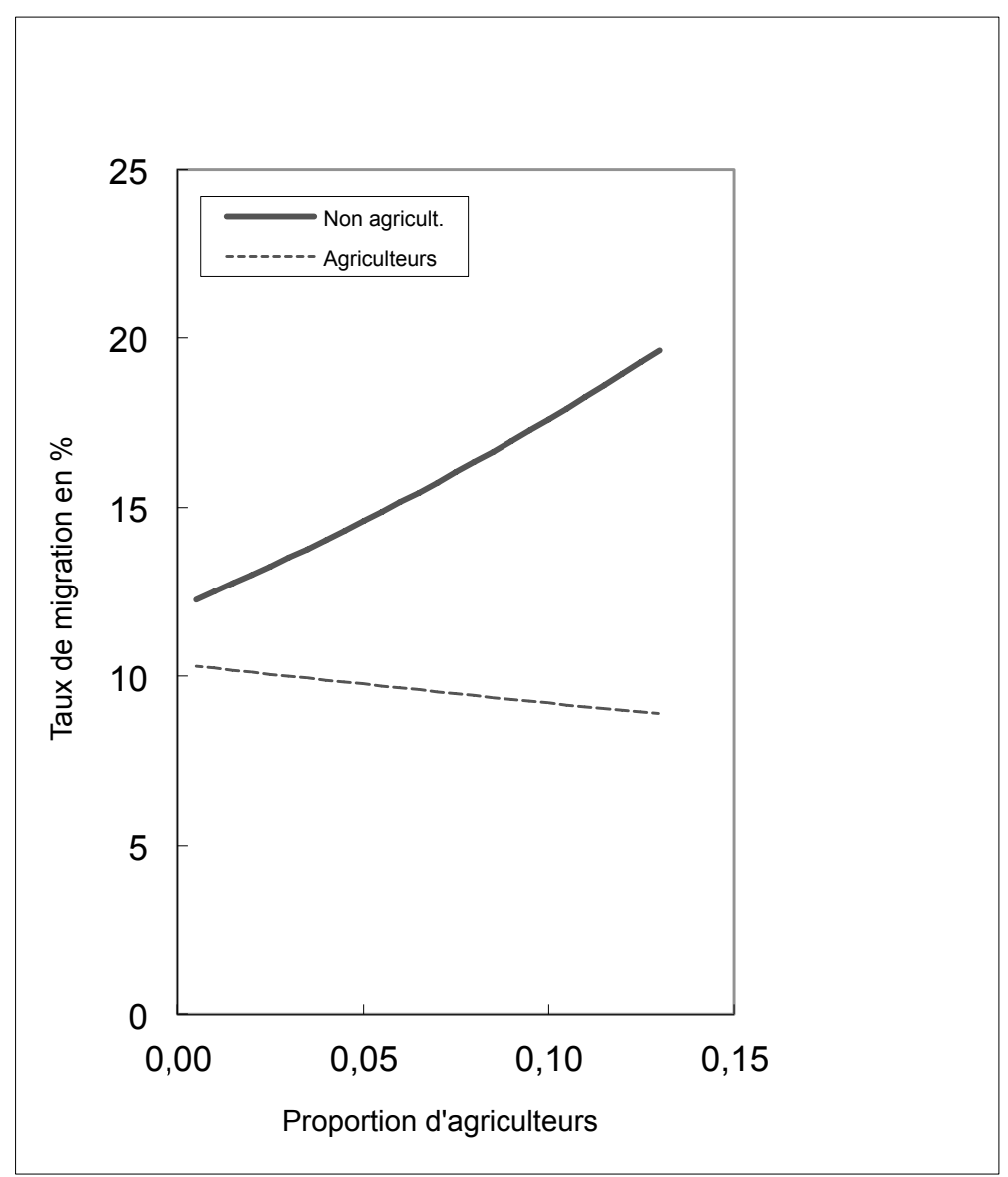

Figure 3. Taux de migration des agriculteurs et des autres

professions en Norvège selon la proportion

d'agriculteurs dans chaque région 
Ce modèle fournit des résultats qui permettent d'harmoniser les résultats contradictoires précédemment obtenus: le fait d'être agriculteur réduit toujours fortement sa probabilité de migrer, qui est pratiquement indépendante de la région. Mais cette fois-ci, l'augmentation des taux d'émigration avec le pourcentage d'agriculteurs s'explique d'une façon tout à fait différente de la précédente. En effet, dans l'analyse des données agrégées, c'est la constance des probabilités d'émigrer des agriculteurs et des autres (à des valeurs différentes), qui, par le jeu des pourcentages d'agriculteurs, explique que c'est dans les régions où il y a plus d'agriculteurs que les taux d'émigration sont plus élevés. Ici, c'est la variation de ces probabilités d'émigrer des non agriculteurs selon le pourcentage d'agriculteurs présents dans la région qui vient expliquer le même résultat.

Extrait : Du groupe à l'individu. Synthèse multiniveau. Éditions de l'Ined/PUF, 2004. 